\title{
CD133 Expression in Colon Cancer. An Immunohistochemical Analysis of 72 Patients
}

\author{
Miana Gabriela Pop ${ }^{1 *}$, Ana Maria Fiț ${ }^{2}$, Dana Bartoș ${ }^{1,3}$, Adrian Bartoș, Ștefan Cristian Vesa ${ }^{5}$, Cosmin \\ Puia $^{4}$, Aj-Hajjar Nadim ${ }^{4}$ and Iancu Cornel ${ }^{3}$ \\ ${ }^{1}$ Department of Anatomy and Embriology, Romania \\ ${ }^{2}$ Deptartment of Anatomical Pathology, Romania \\ ${ }^{3}$ Regional Institute of Gastroenterology and Hepatology, Romania \\ ${ }^{4}$ Surgical Department, Romania \\ ${ }^{5}$ Deptartment of Pharmacology, Toxicology and Clinical Pharmacology, Romania \\ *Corresponding author: Miana Gabriela Pop, University Assistant, Department of Anatomy and Embriology, Iuliu Hatieganu \\ University of Medicine and Pharmacy, Cluj-Napoca, Romania
}

\section{ARTICLE INFO}

Received: 幽 October 28, 2019

Published:

Citation: Miana Gabriela Pop, Ana Maria Fiț, Dana Bartoș, Adrian Bartoș, Ștefan Cristian Vesa, et al. CD133 Expression in Colon Cancer. An Immunohistochemical Analysis of 72 Patients. Biomed J Sci \& Tech Res 22(5)-2019. BJSTR. MS.ID.003801.

Keywords: Colon; Cancer; Tumor; CD133; Immunohistochemistry

\section{ABSTRACT}

Objectives: The aim of this study was to analyse CD133 expression in colon cancer samples, to evaluate the correlation between CD133 expression in colon cancer tissue and the clinical and pathological characteristics of the patients and to evaluate its prognostic role in OS. CD133 is a transmembrane protein of $120 \mathrm{kDa}$ whose expression was confirmed in various digestive and non-digestive cancers. In colon cancer, studies found CD133 expression a negative prognostic marker for advanced stages of the disease and Overall Survival (OS).

Methods: The study included patients diagnosed with colon cancer between January 2018 - December 2018 that undergone surgical treatment. CD133 expression was analyzed in tumor samples through immunohistochemical detection, using the anti-CD133 polyclonal antibody.

Results: CD133 expression was confirmed at tumoral level: $51 / 72,70.8 \%$ of the cases had CD 133 expression (32/72, 44.4\% had grade 2 CD133 expression while 19/72, $26.4 \%$ had grade 1 CD133 expression; $21 / 72$, 29.1\% of the samples had no CD133 expression (grade 0). The relation between CD133 expression at tumoral level and the clinical and pathological characteristics of the patients did not found, however, any significant correlation $(\mathrm{p}<0.05)$. No relation was observed between CD133 expression and 1-year OS of colon cancer patients included in the study.

Conclusion: CD133 expression was confirmed in colon cancer tissue but its role as a prognostic marker for advanced stages of the disease or poor OS was not highlighted.

\section{Introduction}

Various biomarkers were used for Cancer Stem Cells (CSCs) identification in colon cancer patients: CD133, EpCAM (epithelial intracellular adhesion molecule)/CD326, CD44, CD24, or CD166 [1]. Cancer stem cells (CSCs) involvement in tumor mass formation, in cancer progression, in recurrence and, most important, in resistance to chemotherapy is acknowledg, likewise their major role in carcinogenesis and metastatic processes. CD133 or prominin-1 is a glycoprotein of $120 \mathrm{kDa}$ encoded by the gene PROM1 positioned on the short arm of chromosome 4 [2]. Studies conducted so far in colon cancer found that high CD133 expression at tumoral level correlates with advanced stages of the disease and poor overall survival (OS) [3-5], supporting its use as a negative prognostic factor. The aim of this study was (1) to analyze CD133 expression in colon cancer samples, (2) to evaluate if CD133 expression 
correlates with either clinical or pathological characteristics of the neoplastic patients and (3) to test its prognostic value regarding Overall Survival (OS) of colon cancer patients.

\section{Material and Methods}

\section{Patients and Data Collection}

This is a prospective, longitudinal, observational, analytical cohort study performed on 72 patients diagnosed with colon cancer between January - December 2017 in the Surgical Department of the Regional Institute of Gastroenterology and Hepatology Prof. Dr. Octavian Fodor from Cluj-Napoca, Romania. Patients under the age of 18 , those with rectal cancer or operated with palliative intent (internal diversions or colostomies) were excluded from the study. The research protocol was approved by the Ethics Committee of the Institute. The following variables were registered in an Excel database: demographic data (age, sex), associated comorbidities (type II diabetes mellitus, cardiovascular disease, pulmonary disease, hepatic disease, renal disease, gynecological disease, neurological pathology, endocrine pathology, associated neoplasms), tumor-related data (location of the tumor - ascending, transverse, descending or sigmoid colon, size of the tumorbelow $3 \mathrm{~cm}$, between 3 and $5 \mathrm{~cm}$ and over $5 \mathrm{~cm}$ in diameter, stage of the disease TNM, Tumor Wall Involvement (T), Lymph Node Involvement $(\mathrm{N})$, presence or absence of distant Metastases (M), Lymphatic (L) and Perivascular (V) invasion histopathological type of the tumor and the degree of tumor differentiation - G1 or well differentiated tumors, G2 or moderate differentiated tumors, G3 or poor differentiated tumors, presence of invasion in surrounding organs), biological data (leukocyte level 4000-10000/ $\mu \mathrm{L}$, serum hemoglobin 14-18g/dl, blood platelet count $140000-440000 / \mu \mathrm{L}$, serum total proteins $6-8 \mathrm{~g} / \mathrm{dl}$ and albumins $3.5-5.2 \mathrm{~g} / \mathrm{dl}$, serum liver transaminases GOT 5-45 U/l, GPT 5-45 U/l, glucose level 70-115 $\mathrm{mg} / \mathrm{dl}$, total bilirubin levels $0.1-1.2 \mathrm{mg} / \mathrm{dl}$, pancreatic amylases 30 $100 \mathrm{U} / \mathrm{l})$ and carcinoembryonic antigen level $(<3 \mathrm{ng} / \mathrm{ml})$.

\section{Tissue Harvesting Protocol}

From each patient subjected to colon cancer surgery included in the study, a tissue sample of $2-3 \mathrm{~mm}$ from the tumor was harvested after resection of the colonic segment, all the resections being performed in accordance to oncological principles. Each sample was fixed in formaldehyde $4 \%$ solution and transferred to the Anatomopathological Department for paraffin section procession and preservation. Blocked tissue sections were stored at $4{ }^{\circ} \mathrm{C}$ for the night. The specimen tissue were dewaxed and incubated with hydrogen peroxide solution for 15 minutes. After antigen retrieval, tissue fragments were labeled with primary anti-CD133, clone ab19898 specific antibody. Tissue sections were incubated at $4^{\circ} \mathrm{C}$ in a 1:100 dilution, washed with PBS and incubated for 30 minutes before staining with DBA. Staining of the tumor cell membrane was considered for scoring. For each tumor sample, staining percentage of tumor cells expressing CD133 was analyzed. Results were grouped as Grade 0 (zero) CD133 in case of total absence of staining, Grade 1 CD133 if $<50 \%$ of cells express CD133 and Grade 2 CD133 if more than $50 \%$ of cells express CD133. All cytoplasmic and membranous staining were scored. Results corresponding to each patient were further recorded in the Excel database in order to be subjected to statistical analysis.

\section{Statistical Analysis}

Medcalc Statistical Software version 17.9.7 (MedCalc Software, Ostend, Belgium; http://www.medcalc.org; 2017) was used for statistical analysis. Categorical data were expressed as frequency and percentage while continuous variables were expressed as median and Standard Deviation (SD). The correlation between variables were analysed using Spearman's rho. A p value $<0.05$ was considered statistically significant.

\section{Results}

From a total number of 72 patients, an equal percentage, 36 (50\%) of male and female sex patients were included in the study. Mean age was $64.5 \pm 7.8$ years with range $60-69.75$. Most of the tumors were adenocarinoma, 66/72 (91.7\%) while 6/72 (8.3\%) were characaterized by a mucinos pattern. CD133 expression was not significant different between these two histological types. Most of the patients were diagnosed with stage II, 30/72 (41.7\%), respectively stage III, 27/72 (37.5\%) colon cancer. Only $4 / 72(5.6 \%)$ of cases were stage I and a number of $11 / 72(15.2 \%)$ patients presented with distant metastases (stage IV disease). Regarding the analyzed marker, the disease stage didn't have a significant difference in CD133 expression. The sigmoid, 36/72 (50\%) and ascending colon, 28/72 (38.9\%) were the sites most commonly affected by the tumoral process. Only $5 / 72(6.9 \%)$ and $3 / 72(4.2 \%)$ of tumors were located in transverse and descending colon. No significant difference was seen between tumor site and CD133 expression. The size of the tumor ranged from 1 to $10 \mathrm{~cm}$ with a mean size of $4.77 \pm 1.42 \mathrm{~cm}$. The relation between tumor size and CD133 expression was not statistical significant ( $p>0.05)$. A moderate differentiated pattern (G2) was seen in the majority of the patients, 57/72 (79.2\%). CD133 expression was independent from the grading of the tumor.

CD133 expression was positive in 51/72, 70.8\% cases while 21/72, 29.1\% samples had no CD133 expression. Grade 2 CD133 ( $>50 \%$ of cells expressing CD133) was observed in $32 / 72,44.44 \%$ of all cases, while grade 1 ( $<50 \%$ of cells expressing CD133) was found in 19/72, 26.4\% patients. Examples of negative and positive colon cancer samples for CD133 can be seen in (Figure 1) respectively (Figure 2) Univariate analysis of the clinical and pathological parameters of the patients in relation to CD133 expression was further performed. According to the results obtained, tumour size $(p=0.03)$ and the mucinous variant of colon cancer $(p=0.05)$ reached statistical significance $(\mathrm{p}<0.05)$ (Table 1$)$. The other analysed variables did not take statistical significance $(p>0.05)$. (data not shown). Variables that achieved statistical significance in univariate analysis were further introduced in a logistic regression 
multivariate analysis. None of the variables achieved, however, statistical significance ( $p>0.05)$ (data not shown). Furthermore, the prognostic value of CD133 regarding overall survival (OS) in patients operated for colon cancer was tested. Overall survival of the patients included in the study was not influenced by CD133 expression at tumoral level (no significant difference was seen between CD133 expression at tumor level and 1-year survival) $(p>0.05)$.

Table 1: Univariate analysis of relevant clinical and pathological factors in relation to CD133 expression in colon cancer samples.

\begin{tabular}{|c|c|c|c|c|}
\hline \multicolumn{2}{|c|}{ Variables } & $\begin{array}{c}\text { Grade 0 CD133 } \\
\text { n (\%) }\end{array}$ & $\begin{array}{c}\text { Grade 1-2 } \\
\text { CD133 n (\%) }\end{array}$ & p \\
\hline \multirow{2}{*}{$\begin{array}{c}\text { Mucinos } \\
\text { adenocarci- } \\
\text { noma }\end{array}$} & No & $17(81)$ & $49(96.1)$ & \multirow{2}{*}{0.05} \\
\cline { 2 - 4 } & Yes & $4(19)$ & $2(3.9)$ & \\
\hline \multirow{2}{*}{$\begin{array}{c}\text { Tumor size } \\
\text { (cm) }\end{array}$} & $<3$ & $3(14.3)$ & $7(13.7)$ & \\
\cline { 2 - 4 } & 5 -Mar & $3(14.3)$ & $23(45.1)$ & 0.03 \\
\cline { 2 - 4 } & $>5$ & $15(71.4)$ & $21(41.2)$ & \\
\hline
\end{tabular}
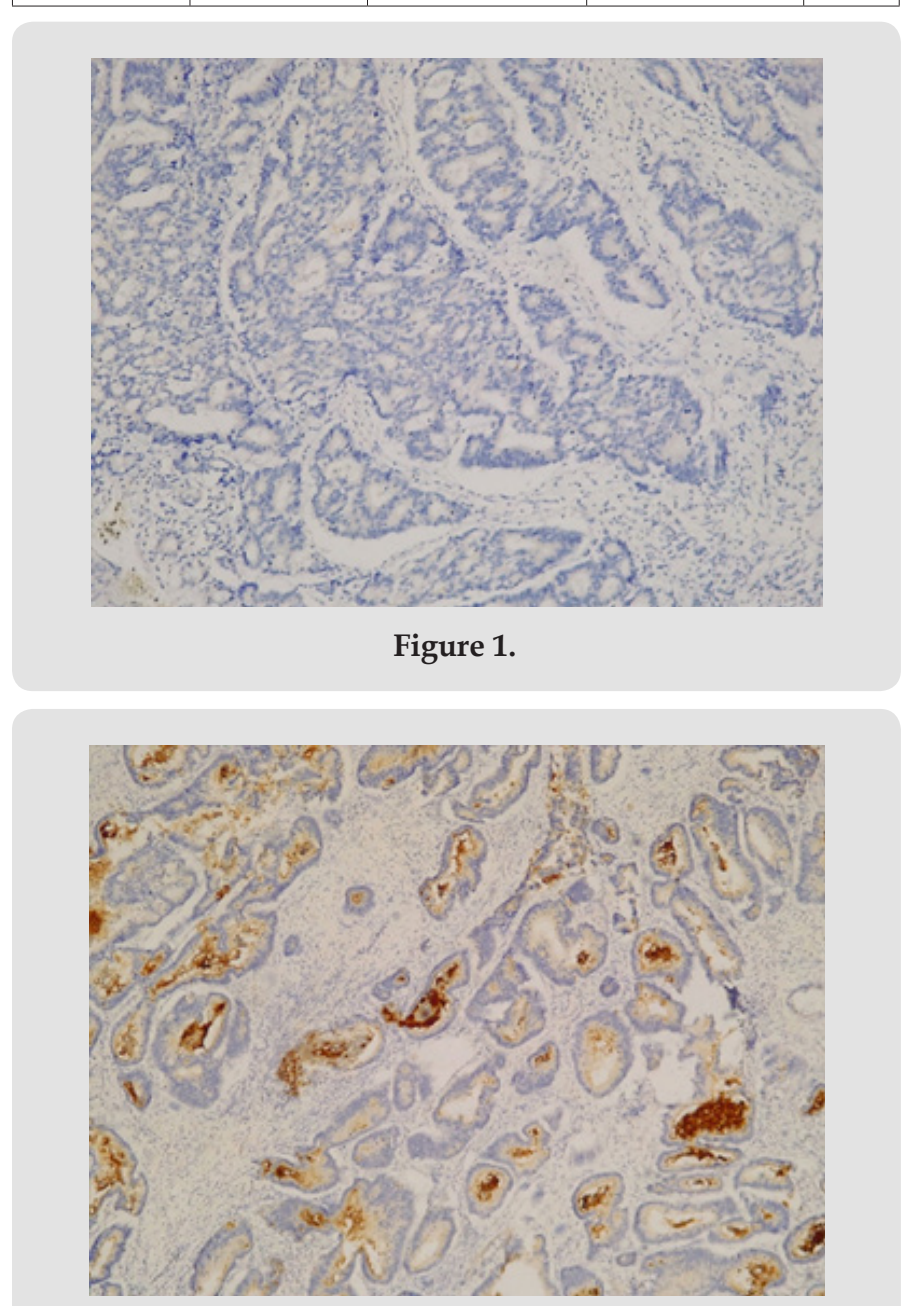

Figure 2.

\section{Discussion}

Colon cancer survival decreases substantially when distant organ metastases occur. Therefore, a crucial step in the oncological treatment of colon cancer patients is based on metastases prevention. Tumor mass is constituted by a heterogenous group of cells, among which are individualized cells characterized by invasiveness and differentiation properties. These cells are called Cancer Stem Cells (CSCs) and are thought to be responsible for initiation of the metastatic process. Identification of CSCs at tumor level, alongside with evaluation of their function represent thus a subject of interest in recent literature. CSCs detection is currently possible through identification of various Cluster of Differentiation (CD) on both the surface of the cellular membrane and in the cytoplasm of tumor cells. Such examples are: CD133, CD24, CD29, CD44, EpCAM (epithelial intracellular adhesion molecule), Lgr5, CD166, ALDH1A1, ALDH1B1 [6].

Identification of various $\mathrm{CD}$ at tumor level was previously confirmed, as their function in cell-cell, cell-matrix interaction and solid tumor mass development is known, but besides this phenomenon, little is known about their behaviour, despite the large number of studies that currently exist in literature. Of these, the most important tool in CSCs identification is considered to be CD133 or prominin-1 [7]. Its expression was evaluated and confirmed in various digestive (colorectal, pancreatic, liver) and non-digestive (prostate, kidney, ovarian) cancers [7-8]. CD133 acts as a contributor in Wnt/ß-catenin pathway of colon cancer, stimulating tumor proliferation [9]. However, the prognostic role of CD133 in advanced colon cancer has not been fully elucidated. While some studies found a significant correlation between CD133 expression at tumor level and tumor size [8], degree of differentiation, presence of distant metastasese [10,11], degree of wall involvement (T), Peri-Lymphatic (L) and peri-venous invasion [12], others did not find the abovementioned association [13]. CD133 expression and Overall Survival (OS) of colon cancer patients was analyzed but heterogenous results were found.

Two meta-analysis found a significant correlation between CD133 expression at tumor level and OS $[3,14]$. However, the association was not confirmed by other research groups [10,13], similar to the results obtained in the present study. Even if CD133 presence in colon CSCs is admitted, its role in cancer progression remains unclear. The countless variants of anti-CD133 antibodies used for CSCs identification (via CD133) alongside with the heterogenicity in CD133 staining reports (as intensity, percentage or a product of these two) have led to confusion and disparate results regarding its role in colorectal carcinogenesis. In the present study, CD133 was confirmed in colon cancer samples, aspect that supports its assessment as a therapeutic target and its involvement in solid tumor mass formation due to its cell-cell and cell-matrix adhesion. However, CD133 usage as a prognostic factor for advanced stages of disease or OS is questioned and remains a subject of debate.

\section{Conclusions}

a) CD133 is expressed in colon cancer patients. The majority of the analyzed patients in this study were characterized by positive CD133 expression. 
b) According to the results of the study, CD133 expression cannot be used as a prognostic marker for advanced colon cancer stages and should rather be tested as a molecular target.

c) CD133 expression at tumor level cannot be used as a predictive biomarker for 1-year overall survival.

Study Limitations: The sample of the study is small; large scale analysis could be necessary in order to confirm this result.

Disclosure Statement: The authors report no declarations of interest.

Funding Details: This work was supported by the University of Medicine and Pharmacy "Iuliu Hatieganu" Cluj-Napoca, Romania under the research grant number 7690/55/15.04.2016 used for the funding of this study.

\section{Conflict of Interest}

The authors have no conflict of interest to declare.

\section{References}

1. Glumac PM, LeBeau AM (2018) The role of CD133 in cancer: A concise review. Clin Transl Med 7: 18.

2. Thamm K, Graupner S, Werner C, Huttner WB (2016) Monoclonal Antibodies 13A4 and AC133 do not recognize the canine ortholog of mouse and human stem cell antigen prominin-1 (CD133). PLoS ONE 11(10): e0164079.

3. Chen S, Song X, Chen Z, Li X, Li M, et al. (2013) CD133 expression and the prognosis of colorectal cancer: a systematic review and metaanalysis. PLoS ONE 8(2): e56380.

4. Fang C, Fan C, Wang C, Huang Q, Meng W, et al. (2017) Prognostic value of CD133(+) CD54(+) CD44(+) circulating tumor cells in colorectal cancer with liver metastasis. Cancer Med 6(12): 2850-2857.

\section{ISSN: 2574-1241}

DOI: 10.26717/BJSTR.2019.22.003801

Miana Gabriela Pop. Biomed J Sci \& Tech Res

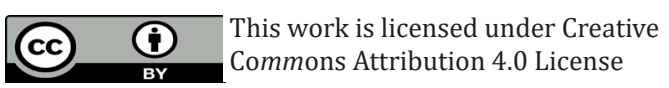

Submission Link: https://biomedres.us/submit-manuscript.php
5. Wang BB, Li ZJ, Zhang FF, Hou HT, Yu JK, (2016) Clinical significance of stem cell marker CD133 expression in colorectal cancer. Histol Histopathol 31(3): 299-306.

6. Pop MG (2019) Stem Cell Markers in Colon Cancer in MG Pop. Basic Principles and Practice in General Surgery.

7. Kazama S, Kishikawa J, Kiyomatsu T, Kawai K, Nozawa $H$, et al. (2018) Expression of the stem cell marker CD133 is retaled to tumor development in colorectal carcinogensis. Asian Jouranl of Surgey 41(3): 274-278.

8. Ren F, Sheng WQ Du X (203) CD133: A cancer stem cell marker, is used in colorectal cancers. World J Gastroenterol 19: 2603-2611.

9. Barzegar Behrooz A, Syahir A, Ahmad S (2018) CD133: beyond a cancer stem cell biomarker. J Drug Target 27: 1-13.

10. Nosrati A, Naghshvar F, Maleki I, Salehi F (2016) Cancer stem cells CD133 and CD24 in colorectal cancers in Northern Iran. Gastroenterology and Hepatology from Bed to Bench 9(2): 132-139.

11. Sahlberg SH, Spielberg D, Glimelius B, Stenerlow B, Nestor M (2014) Evaluation of cancer stem cell markers CD133, CD44, CD24: Association with AKT isoforms and radiation resistance in colon cancer cells. PLoS One 23(9): e94621

12. Huang R, Mo D, Wu J, Ai H, Lu Y (2018) CD133 expression correlates with clinicopathologic features and poor prognosis of colorectal cancer patients: An updated meta-analysis of 37 studies. Medicine 97(23): e10446.

13. Pitule P, Miroslava C, Daum 0, Vojtisek J, Vycital 0 , et al. (2014) Immunohistochemical detection of cancer stem cell related markers CD44 and CD 133 in metastatic colorectal cancer patients. BioMed Research International 2014: 7.

14.Wang K, Xu J, Zhang J, Huang J (2012) Prognostic role of CD133 expression in colorectal cancer: A meta-analysis. BMC Cancer 12: 53.

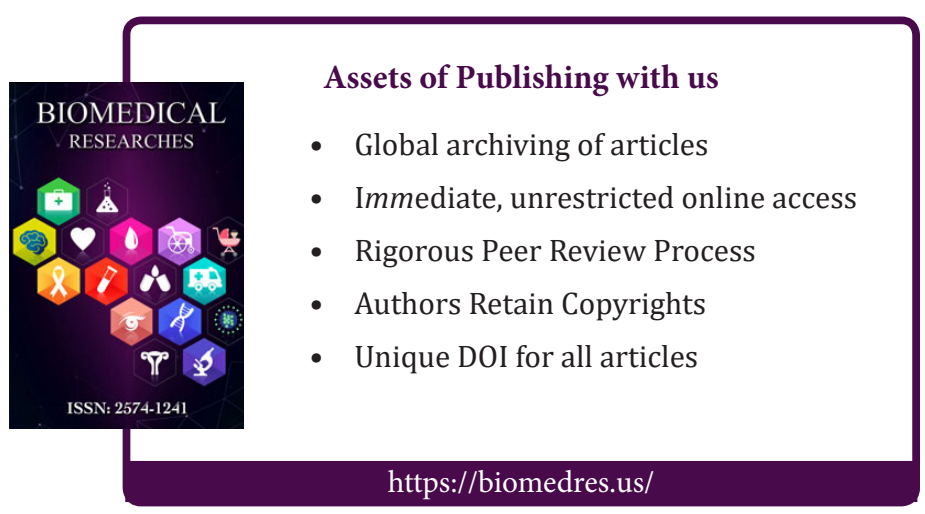

